\title{
Novel antiviral properties of azithromycin in cystic fibrosis airway epithelial cells
}

\author{
Aline Schögler ${ }^{1,2,3}$, Brigitte S. Kopf ${ }^{1,2}$, Michael R. Edwards ${ }^{4}$, \\ Sebastian L. Johnston ${ }^{4}$, Carmen Casaulta ${ }^{1}$, Elisabeth Kieninger ${ }^{1}$, \\ Andreas Jung ${ }^{5}$, Alexander Moeller ${ }^{5}$, Thomas Geiser ${ }^{2,6}$, Nicolas Regamey ${ }^{1,2,7}$ \\ and Marco P. Alves ${ }^{1,2,7}$
}

\begin{abstract}
Affiliations: 'Division of Paediatric Respiratory Medicine, University Children's Hospital, Berne, Switzerland. ${ }^{2}$ Dept of Clinical Research, University of Berne, Berne, Switzerland. ${ }^{3}$ Graduate School for Cellular and Biomedical Sciences, University of Berne, Berne, Switzerland. ${ }^{4}$ Airway Disease Infection Section, MRC and Asthma UK Centre in Allergic Mechanisms of Asthma and Centre for Respiratory Infection, National Heart and Lung Institute, Imperial College London, London, UK. 'Division of Respiratory Medicine, University Children's Hospital, Zurich, Switzerland. 'Dept of Pulmonary Medicine, University Hospital Berne, Berne, Switzerland.

${ }^{7}$ These authors contributed equally.
\end{abstract}

Correspondence: Nicolas Regamey, Dept of Clinical Research, University of Berne, 3008 Berne, Switzerland. E-mail: Nicolas.Regamey@dkf.unibe.ch

ABSTRACT Virus-associated pulmonary exacerbations, often associated with rhinoviruses (RVs), contribute to cystic fibrosis (CF) morbidity. Currently, there are only a few therapeutic options to treat virus-induced CF pulmonary exacerbations. The macrolide antibiotic azithromycin has antiviral properties in human bronchial epithelial cells. We investigated the potential of azithromycin to induce antiviral mechanisms in CF bronchial epithelial cells.

Primary bronchial epithelial cells from CF and control children were infected with RV after azithromycin pre-treatment. Viral RNA, interferon (IFN), IFN-stimulated gene and pattern recognition receptor expression were measured by real-time quantitative PCR. Live virus shedding was assessed by assaying the $50 \%$ tissue culture infective dose. Pro-inflammatory cytokine and IFN- $\beta$ production were evaluated by ELISA. Cell death was investigated by flow cytometry.

RV replication was increased in CF compared with control cells. Azithromycin reduced RV replication seven-fold in CF cells without inducing cell death. Furthermore, azithromycin increased RV-induced pattern recognition receptor, IFN and IFN-stimulated gene mRNA levels. While stimulating antiviral responses, azithromycin did not prevent virus-induced pro-inflammatory responses.

Azithromycin pre-treatment reduces RV replication in CF bronchial epithelial cells, possibly through the amplification of the antiviral response mediated by the IFN pathway. Clinical studies are needed to elucidate the potential of azithromycin in the management and prevention of RV-induced CF pulmonary exacerbations.

@ERSpublications

Azithromycin reduces rhinovirus load in CF bronchial cells, possibly through the induction of the interferon pathway http://ow.ly/BVw2U

This article has supplementary material available from erj.ersjournals.com

Received: June 042014 | Accepted after revision: Sept 022014 | First published online: Oct 302014

Support statement: This study was supported by a financial grant from the Mukoviszidose Institut gGmbH, Bonn, Germany, the research and development arm of the German Cystic Fibrosis Association Mukoviszidose e.V.

Conflict of interest: Disclosures can be found alongside the online version of this article at erj.ersjournals.com

Copyright OERS 2015 


\section{Introduction}

Viruses targeting the respiratory tract have a significant clinical impact on cystic fibrosis (CF) lung disease. It is estimated that $31-61 \%$ of pulmonary exacerbations in CF patients are triggered by respiratory viruses $[1,2]$. The most predominant viruses exacerbating CF lung disease are human rhinoviruses (RVs), which are detected in up to $70 \%$ of virus-induced CF exacerbations [3, 4]. There are, to date, only a few therapeutic options to treat or prevent virus-induced CF exacerbations. The current management consists of the seasonal influenza vaccination and passive immunoprophylaxis against respiratory syncytial virus infection, which is recommended in certain countries [5]. Specific antiviral treatments against influenza are available as therapeutic strategies and prescribed to CF patients [6]. However, there are no available treatments against RV infections.

Azithromycin is a macrolide antibiotic widely used for the treatment of CF patients colonised with Pseudomonas aeruginosa [7, 8]. Beyond their well-established antibacterial effects, a novel and potentially promising property of macrolides is the inhibition of respiratory viral infections in vitro in healthy airway epithelial cells [9-12]. Azithromycin potentially works by stimulating the host antiviral responses through the induction of interferons (IFNs) and IFN-stimulated genes (ISGs) [12].

The airway epithelium is central in the antiviral defence of the lung by producing IFNs upon infection with RVs, leading to the induction of ISGs that directly interfere with virus replication $[13,14]$. In vitro and in vivo, type I IFN is important in the host defence against RV, with pre-treatment of endogenous IFN robustly reducing virus load in in vitro cell culture models [15-17]. This highlights the potential importance of IFNs as therapies to control RV replication in the human airways and highlights the potential of IFN inducers such as azithromycin as treatments for virus-associated CF exacerbations. Consequently, we used an in vitro model based on primary paediatric CF bronchial epithelial cells to evaluate the antiviral activity of azithromycin towards RV infection.

\section{Material and methods}

Study participants

CF subjects and healthy volunteers were recruited at the University Hospitals of Berne and Zurich, Switzerland, and bronchial epithelial cells successfully cultured from $11 \mathrm{CF}$ and 14 control children (median (range) age 8.21 (1.14-14.98) years and 9.47 (1.36-16.19) years, respectively). Clinical characteristics of the study participants are shown in table 1. Exclusion criteria were bleeding tendency, therapy with anticoagulants and/or immunosuppressive agents, steroid use within the past 3 months and atopy (the two latter for controls only). As no mutation was found for patient number nine, CF diagnosis was made using the following criteria: chronic cough, fixed bronchial obstruction and two independent sweat tests with chloride $>60 \mathrm{mmol} \cdot \mathrm{L}^{-1}$. The ethics committees of the Canton of Berne and Zurich, Switzerland, gave approval for the study and written informed consent was obtained from all study participants and/or caregivers.

\section{Cell culture and virus}

Primary bronchial epithelial cells were obtained from CF and control children by brushing the bronchial tree during a clinically indicated bronchoscopy or through an endotracheal tube during an elective surgery under general anaesthesia [19]. Except from one CF patient, bronchial cells were obtained during routine surveillance bronchoscopy and were therefore taken at a stable stage of the disease (table 1). Cell cultures were set up by seeding bronchial epithelial cells in collagen (Pure Col; Inamed, Gauting, Germany) coated culture flasks (Techno Plastic Products, Trasadingen, Switzerland). The cells were grown in bronchial epithelial growth medium supplemented with SingleQuots (BEGM; Lonza, Basel, Switzerland) and Primocin $\left(100 \mu \mathrm{g} \cdot \mathrm{mL}^{-1}\right.$; InvivoGen, San Diego, CA, USA $)$ in a humidified incubator at $37^{\circ} \mathrm{C}$. CF bronchial cells were additionally treated with amphotericin B $\left(250 \mu \mathrm{g} \cdot \mathrm{mL}^{-1}\right.$; Sigma Aldrich, St Louis, MO, USA) and ceftazidime (100 $\mathrm{g} \cdot \mathrm{mL}^{-1}$; GlaxoSmithKline, Münchenbuchsee, Switzerland) for 5 days after sampling. In order to assess the antiviral activity of azithromycin in bronchial cells, we used an experimental system based on RV1B, as established by others [20]. RV1B stock was propagated and titrated on Ohio-HeLa cells (European Collection of Cell Cultures, Porton Down, UK) as described previously [21].

\section{Azithromycin treatment and virus infection}

At passage 2, the cells were pre-treated with azithromycin dihydrate (50 $\mu \mathrm{M}$; Sigma-Aldrich) and grown in bronchial epithelial basal medium (Lonza) for $24 \mathrm{~h}$. Azithromycin was diluted in $96 \%$ ethanol, at a final concentration of $0.05 \%$ for $50 \mu \mathrm{M}$. The mode of treatment and concentration of azithromycin was chosen based on previous observations by ourselves and others [12], and is in the range of concentrations found in patients under azithromycin treatment [22]. Cells were infected with RV1B at a multiplicity of infection of 4 for $1 \mathrm{~h}$. Supernatants and RNA were harvested at $24 \mathrm{~h}$ post infection. Cells treated with medium alone 
TABLE 1 Demographic and clinical characteristics of study subjects

Controls

CF patients

\begin{tabular}{|c|c|c|c|c|c|c|c|c|c|c|c|c|c|c|c|}
\hline & Sex & $\begin{array}{c}\text { Age } \\
\text { years }\end{array}$ & Atopy ${ }^{\#}$ & $\begin{array}{l}\text { Steroid } \\
\text { use }^{\pi}\end{array}$ & & Sex & $\begin{array}{l}\text { Age } \\
\text { years }\end{array}$ & Atopy ${ }^{\#}$ & $\begin{array}{l}\text { Steroid } \\
\text { use }\end{array}$ & $\begin{array}{c}\text { FEV1 } \\
\% \text { pred }\end{array}$ & $\begin{array}{l}P \text {. aeruginosa } \\
\text { colonisation }^{+}\end{array}$ & Genotype & $\begin{array}{c}\text { Under AZM } \\
\text { treatment }\end{array}$ & $\begin{array}{c}\text { Under } \\
\text { exacerbation } \$\end{array}$ & Microorganisms $^{f}$ \\
\hline 1 & $\mathrm{~F}$ & 6.8 & No & No & 1 & M & 8.2 & No & Inhaled & 101 & No & F508del/R347P & No & No & None \\
\hline 2 & $\mathrm{~F}$ & 11.3 & No & No & 2 & M & 3.8 & No & No & 108 & No & F508del/F508del & No & No & $\begin{array}{l}\text { S. aureus } \\
\text { S. pneumoniae } \\
\text { H. influenzae }\end{array}$ \\
\hline 3 & M & 12.1 & No & No & 3 & $\mathrm{~F}$ & 4.7 & No & No & ND & Yes & F508del/2347delG & No & Yes & $\begin{array}{l}\text { S. pneumoniae } \\
\text { Penicillium spp. }\end{array}$ \\
\hline 4 & $\mathrm{~F}$ & 1.4 & No & No & 4 & $\mathrm{~F}$ & 11 & Yes & No & 67 & No & G542X/2708del13 & Yes & No & $\begin{array}{l}\text { S. aureus } \\
\text { A. fumigatus }\end{array}$ \\
\hline 5 & M & 7.6 & No & No & 5 & M & 8.7 & Yes & Inhaled & 106 & No & F508del/F508del & No & No & S. aureus \\
\hline 6 & M & 12 & No & No & 6 & M & 2.6 & No & Inhaled & ND & Yes & F508del/F508del & No & No & Penicillium spp. \\
\hline 7 & $\mathrm{~F}$ & 14.8 & No & No & 7 & $\mathrm{~F}$ & 9.1 & Yes & No & 81 & No & F508del/F508del & No & No & S. aureus \\
\hline 8 & $M$ & 4.2 & No & No & 8 & $M$ & 1.1 & No & No & ND & No & F508del/F508del & No & No & $\begin{array}{l}\text { S. aureus } \\
\text { H. influenzae } \\
\text { M. catarrhalis }\end{array}$ \\
\hline 9 & M & 1.7 & No & No & 9 & $\mathrm{~F}$ & 15 & Yes & Systemic & 38 & Yes & F508del/F508del & No & No & S. aureus \\
\hline 10 & M & 16.2 & No & No & 10 & M & 6.2 & No & No & 127 & No & F508del/F508del & No & No & None \\
\hline 11 & $\mathrm{~F}$ & 13.3 & No & No & 11 & M & 10.9 & No & No & 62 & No & No mutation found & No & No & None \\
\hline 12 & M & 1.4 & No & No & & & & & & & & & & & \\
\hline 13 & M & 5.5 & No & No & & & & & & & & & & & \\
\hline 14 & $\mathrm{~F}$ & 14.2 & No & No & & & & & & & & & & & \\
\hline
\end{tabular}

CF: cystic fibrosis; FEV1: forced expiratory volume in $1 \mathrm{~s}$; AZM: azithromycin; F: female; M: male; ND: not determined; S. aureus: Staphylococcus aureus; S. pneumoniae: Streptococcus pneumoniae; H. influenzae: Haemophilus influenzae; A. fumigatus: Aspergillus fumigatus; M. catarrhalis: Moraxella catarrhalis. ": history of hay fever, eczema or asthma; ": any treatment with systemic, inhaled or nasal steroids within the previous 3 months; ${ }^{+}$: at least one Pseudomonas aeruginosa-positive oropharyngeal culture during the previous 12 months; ${ }^{\S}$ : defined as by KIENINGER et al. [18]; ${ }^{f}$ : present in bronchoalveolar lavage fluid at time of brushing. 
(infection media) were used as negative control. Polyinosinic:polycytidylic acid (polyI:C) $\left(1 \mu \mathrm{g} \cdot \mathrm{mL}^{-1}\right.$; Sigma Aldrich) served as positive control.

\section{Virus titration}

Supernatants of infected cell cultures were serially diluted in Dulbecco's modified Eagle's medium (Life Technologies, Carlsbad, CA, USA) containing 4\% fetal calf serum (BioConcept, Allschwil, Switzerland) and titrated on Ohio-HeLa cells. We determined the 50\% tissue culture infective dose (TCID50) per mL of RV by using the Spearman-Karber method [12].

\section{Cytotoxicity}

To assess cell viability, CF bronchial cells were collected $24 \mathrm{~h}$ after azithromycin treatment. The cells were treated with annexin V labelled with Alexa Fluor $647\left(1 \mu \mathrm{g} \cdot \mathrm{mL}^{-1}\right.$; Biolegend, San Diego, CA, USA) for $20 \mathrm{~min}$ and with propidium iodide $\left(0.5 \mu \mathrm{g} \cdot \mathrm{mL}^{-1}\right.$; BD Biosciences, San Jose, CA, USA) directly before data acquisition by flow cytometry (LSRII; BD Biosciences) to quantify apoptosis and necrosis, respectively. Freezing of cells for $30 \mathrm{~min}$ was used as positive control for propidium iodide staining and polyI:C treatment $\left(1 \mu \mathrm{g} \cdot \mathrm{mL}^{-1}\right)$ for $24 \mathrm{~h}$ served as positive control for annexin V staining. PolyI:C induces apoptosis through activation of pattern recognition receptors (PRRs) and subsequent IFN signalling [23]. Data analysis was carried out using FlowJo software (Tree Star, Ashland, OR, USA).

\section{RNA extraction and cDNA synthesis}

Total RNA was extracted using the NucleoSpin RNA kit (Macherey-Nagel, Oensingen, Switzerland). We used $200 \mu \mathrm{g}$ total RNA for the reverse transcription (Omniscript RT kit; Qiagen, Hombrechtikon, Switzerland).

\section{Real-time PCR}

Real-time quantitative PCR was performed using primers $(20 \mu \mathrm{M})$ and probes $(5 \mu \mathrm{M})$ for $18 \mathrm{~S}$ ribosomal RNA (rRNA), RV, IFN- $\lambda 2 / 3$, IFN- $\beta$, low-density lipoprotein receptor (LDLR), Toll-like receptor (TLR)3, TLR2, retinoic acid-inducible gene (RIG)-I, melanoma differentiation-associated protein (MDA)5, viperin (virus inhibitory protein, endoplasmic reticulum-associated, IFN inducible), 2',5'-oligoadenylate synthase (OAS), MxA, interleukin (IL)-8, IL-6 and the $10-\mathrm{kDa}$ IFN- $\gamma$-induced protein (IP-10). The DNA sequences of primers and probes are presented in table 2. PCR reactions consisted of $18 \mu \mathrm{L}$ PCR Master Mix (Life Technologies) and $2 \mu \mathrm{L}$ cDNA. PCR reactions were performed on a 7500 Fast Real-Time PCR System (Life Technologies). To quantify mRNA expression levels of endogenous genes, the $\Delta \Delta \mathrm{Ct}$ method was used; mRNA expression levels were normalised to those of $18 \mathrm{~S}$ rRNA. To quantify viral replication, viral RNA copies were interpolated from a standard curve generated by the serial dilution of a plasmid containing the RV cDNA.

\section{ELISA}

IL-8, IL-6, IP-10 and IFN- $\beta$ protein levels were quantified in cell culture supernatants by ELISA using kits for human IL-8, IL-6 and IP-10 (all from R\&D Systems, Minneapolis, MN, USA), and IFN- $\beta$ (PBL InterferonSource, Piscataway, NJ, USA).

\section{Statistical analysis}

Statistical analysis was performed using Prism 5 software (GraphPad Software Inc., La Jolla, CA, USA). Data are presented as median (interquartile range) unless stated otherwise. To determine differences between two groups, nonparametric paired Wilcoxon tests or Mann-Whitney U-tests were used as appropriate. A p-value $<0.05$ was considered statistically significant.

\section{Results}

CF bronchial epithelial cells have an increased susceptibility to RV1B infection

Several studies have reported a hypersusceptibility of the CF airway epithelium to respiratory viruses [18, 20, 28]. To confirm that CF bronchial cells are more susceptible to RV infection than controls, we assessed virus shedding into supernatants at $24 \mathrm{~h}$ post-infection by a TCID50 assay (fig. 1a). We found that the CF bronchial cells released a significant 2.7 -fold higher amount of live virus compared with control cells $(\mathrm{p}=0.001)$. These results were confirmed by determining RV1B replication in CF bronchial cells compared with controls at $24 \mathrm{~h}$ post-infection by qPCR (intracellular viral RNA) (fig. 1b), indicating that qPCR measurements of RV replication can be used as surrogate for virus shedding, as already shown in healthy cells [12]. 
TABLE 2 Primer and probe sequences $\left(5^{\prime} \rightarrow 3^{\prime}\right)$

Target

Sequence

[Ref.]

\section{S rRNA}

Forward primer Reverse primer Probe

RV

Forward primer Reverse primer Probe

IFN- $\lambda 2 / 3$

Forward primer Reverse primer Probe

IFN- $\beta$

Forward primer Reverse primer Probe

LDLR

Forward primer Reverse primer Probe

TLR3

Forward primer Reverse primer Probe

TLR2

Forward primer Reverse primer Probe

RIG-I

Forward primer Reverse primer Probe

MDA5

Forward primer Reverse primer Probe

Viperin

Forward primer Reverse primer Probe

OAS

Forward primer Reverse primer Probe

MxA

Forward primer Reverse primer Probe

IL-8

Forward primer Reverse primer Probe

IL-6

Forward primer Reverse primer Probe

IP-10

Forward primer Reverse primer Probe
CTGCCACATAGCCCAGTTCA

CTGCCACATAGCCCAGTTCA

FAM-TCTCCACAGGAGCTGCAGGCCTTTA-TAMRA

CACGGATACAGAACCTATGG

ACGAACAGTGTCGCCTACTA

FAM-TCAGACAAGATTCATCTAGCACTGGCTGGA-TAMRA

TGACAATGTCTCACCAAGCTCTG

CTCACGCTACTGGGCTTCTTCT

FAM-CTGCCAGCAACGTCG-TAMRA

AAATTAAAGAGTTTTCTCCAGGGTGTT ATTCCGAATGCTTGTGTTTGC FAM-TTTGGCCTCTTTCTGAACAATGTCCAGC-TAMRA

TTTCACTGCTTTCAACTGGTA

TGGAGAGGCTGATGATGAC

FAM-CAAGACCCACACCATCCACAA-TAMRA

CCAAGCCAAAGCAGTTTTCAA

CACATGGATTCCCCAGTCATG

FAM-TTGAAAAAAGAGCAAAGATATTCTGTGCCCGAC-TAMRA

GATTCAGGCACCATGGGAAGT

AGGCCTGAGCTGGAGTTCTG

FAM-GGGATGCTCTTGCTGCCACATTCTCTT-TAMRA

CACAAAGAAGTGTCCTGCTTGGT

AAGCGCATATATTTCATCCAGAATAAG

FAM-CCTGAATCTAACCAGAAGATGAAAGACTCC-TAMRA

CTGACGCTGACCTGGTTGTCT

CCCCGGCGATTTAACTGAT

FAM-CCTCAGTCCTCTCACCACTTTTCA-TAMRA

CAGCACCTGATGGCCTATCAC

CATGAACTGGATGATCAAAGG

FAM-AGGCCAGCAAGCGCATCTCCAG-TAMRA

CTGGCCGTGGCTCTCTTG

CCTTGGCAAAACTGCACCTT

FAM-CAGCCTTCCTGATTTCTGCAGCTCTGTGT-TAMRA

CCAGGAGCCCAGCTATGAAC

CCCAGGGAGAAGGCAACTG

FAM-CCTTCTCCACAAGCGCCTTCGGT-TAMRA

CCATTCTGATTTGCTGCCTTATC

GCAGGTACAGCGTACAGTTCT

FAM-CTGACTCTAAGTGGCATTCAAGGAGTACCTCTCTC-TAMRA

rRNA: ribosomal RNA; RV: rhinovirus; IFN: interferon; LDLR: low-density lipoprotein receptor; TLR: Toll-like receptor; RIG: retinoic acid-inducible gene; MDA: melanoma differentiation-associated protein; viperin: virus inhibitory protein, endoplasmic reticulum-associated, IFN inducible; OAS: 2',5'-oligoadenylate synthase; IL: interleukin; IP-10: 10-kDa IFN- $\gamma$-induced protein; FAM: 6-carboxyfluorescein; TAMRA: tetramethylrhodamine. 

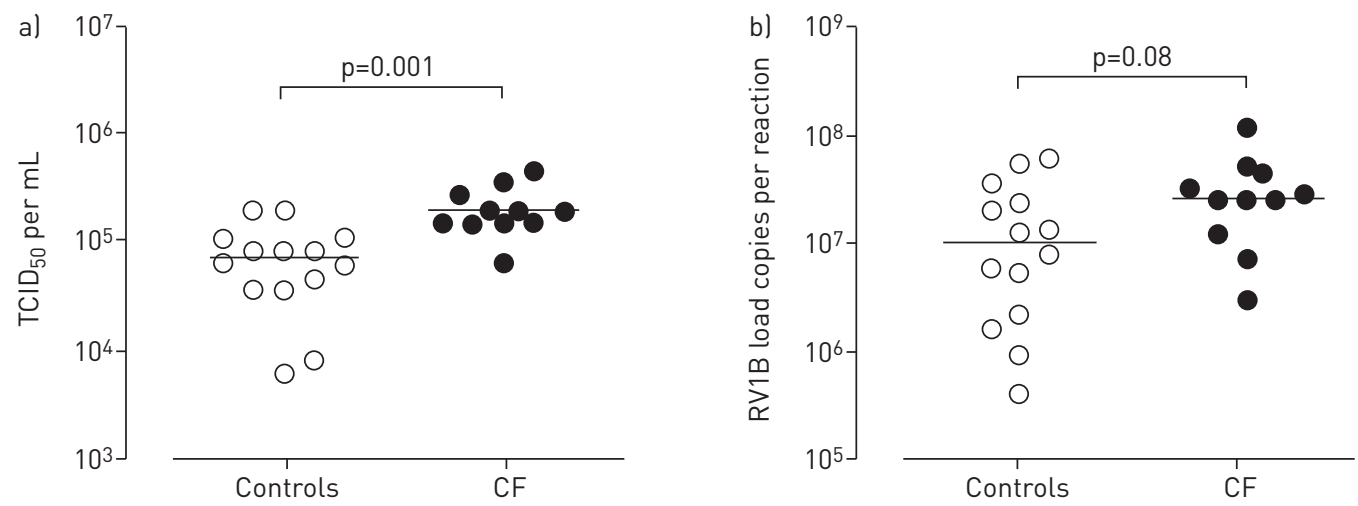

FIGURE 1 Rhinovirus (RV)1B replication is increased in primary cystic fibrosis (CF) bronchial epithelial cells compared with control cells. a) Live RV1B in supernatants was measured by the $50 \%$ tissue culture infective dose (TCID50) assay and b) intracellular viral RNA was assessed in cell lysates by real-time quantitative PCR at $24 \mathrm{~h}$ post-infection in primary control and CF bronchial epithelial cells. Data are presented as median of 11-14 independent experiments. Statistical analysis was performed with the nonparametric Mann-Whitney U-test.

\section{Azithromycin reduces RV1B replication in CF and control bronchial epithelial cells}

To investigate whether azithromycin has antiviral properties in CF and control bronchial cells, RV1B replication after azithromycin treatment was assessed at $24 \mathrm{~h}$ post-infection by qPCR in CF and control bronchial cells. We found that RV1B replication was seven-fold reduced by azithromycin treatment in CF bronchial cells compared with untreated cells $(\mathrm{p}=0.001$; fig. $2 \mathrm{a})$. Similarly, we observed that azithromycin treatment reduced RV1B load nine-fold in control bronchial cells compared with untreated cells ( $p=0.0002$; fig. 2b). Also, azithromycin treatment significantly lowered RV1B replication in nasal CF cells 4.8 -fold in comparison with untreated cells $(\mathrm{p}=0.03$; online supplementary material).

\section{Reduced RV1B replication is not due to azithromycin-induced cytotoxicity in CF bronchial epithelial cells}

We further investigated whether the observed reduced RV1B replication after azithromycin treatment was due to azithromycin-induced cytotoxicity. We therefore assessed cell death with an annexin V/propidium iodide assay by flow cytometry in either azithromycin-treated or -untreated CF bronchial cells. We found that azithromycin treatment did not increase the percentage of annexin V-positive cells compared with untreated cells (fig. 3a, c and e). Similarly, azithromycin treatment did not induce necrosis, as demonstrated by a similar percentage of propidium iodide-positive cells in azithromycin-treated and -untreated CF cells (fig. 3b, d and f).
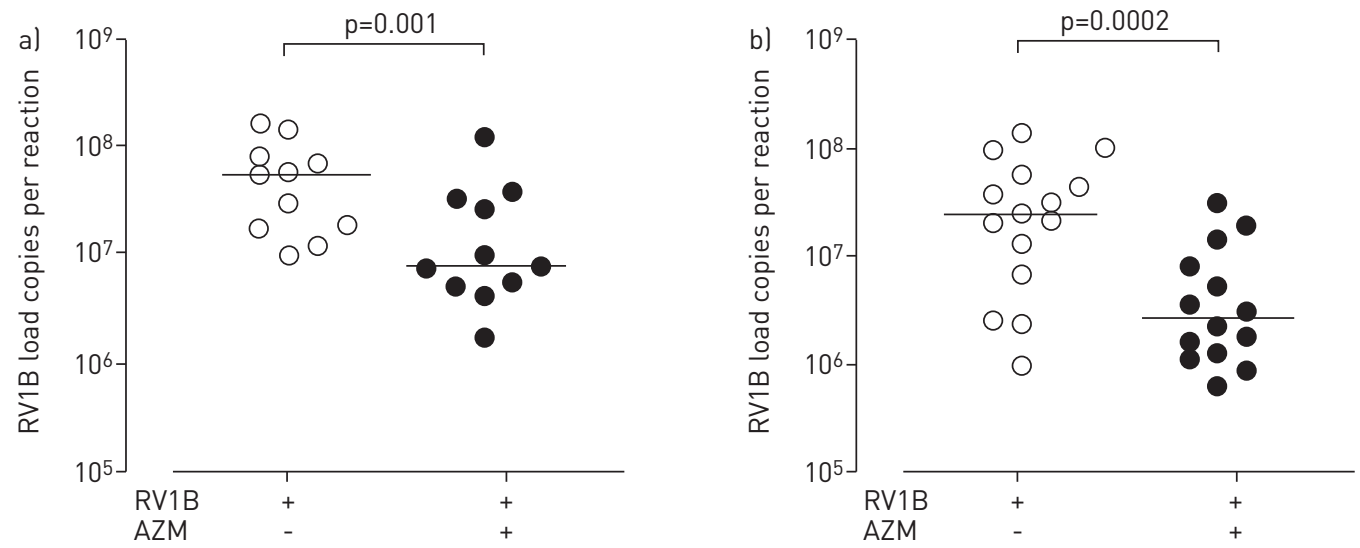

FIGURE 2 Azithromycin (AZM) treatment decreases rhinovirus (RV)1B replication in primary cystic fibrosis (CF) and control bronchial epithelial cells. Intracellular viral RNA was measured at $24 \mathrm{~h}$ post-infection by real-time quantitative PCR in primary a) CF and b) control bronchial epithelial cells with or without pre-treatment with AZM (50 $\mu \mathrm{M})$ for $24 \mathrm{~h}$. Data are presented as median of 11-14 independent experiments. Statistical analysis was performed with the paired nonparametric Wilcoxon rank test. 

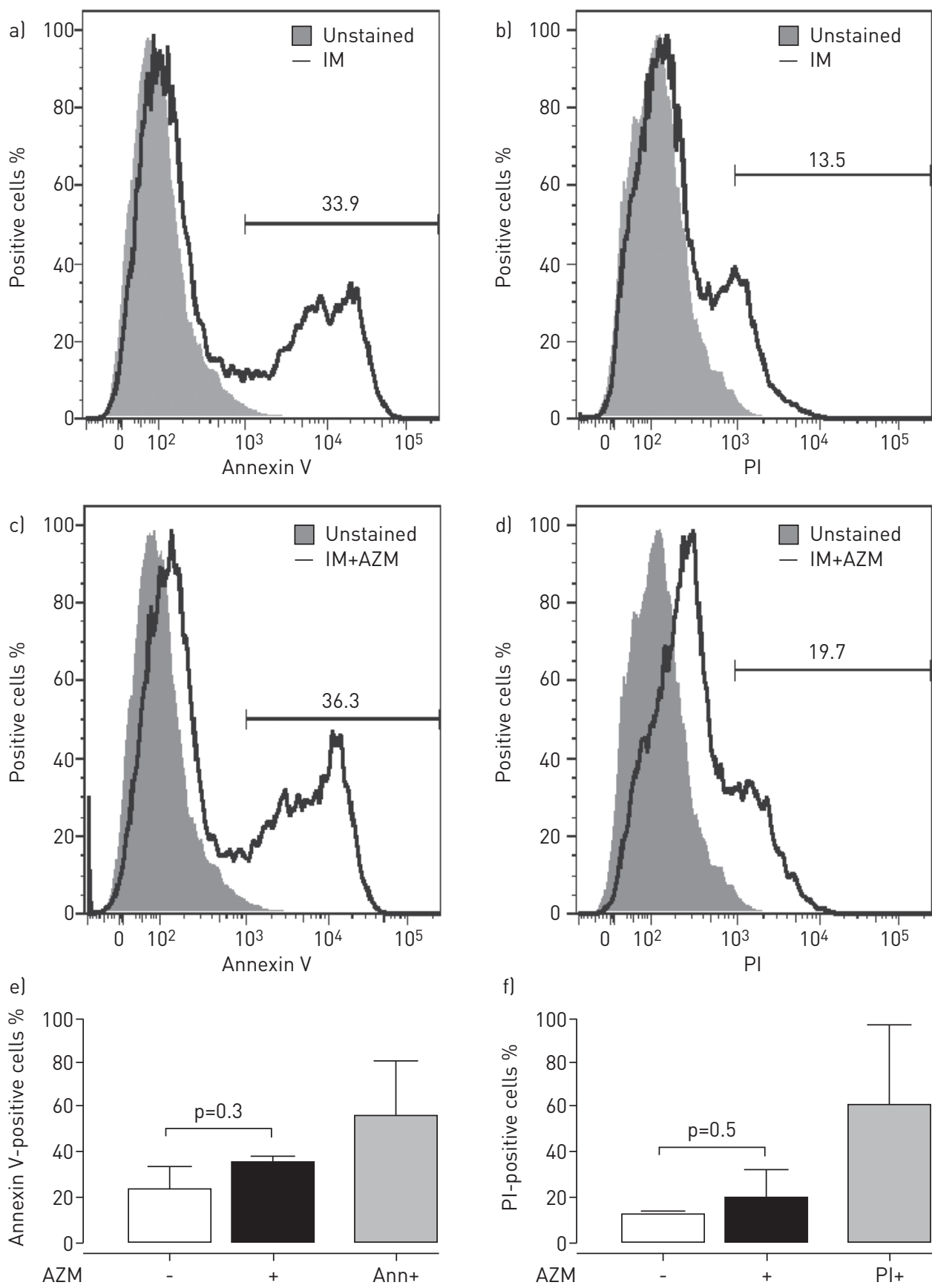

FIGURE 3 Azithromycin (AZM) does not induce cell cytotoxicity in primary cystic fibrosis (CF) bronchial epithelial cells. $\mathrm{a}-\mathrm{d})$ Histograms of annexin $\mathrm{V}$ - (a and c) and propidium iodide (PI)-positive (b and d) primary CF bronchial epithelial cells at $24 \mathrm{~h}$ after treatment with or without AZM $(50 \mu \mathrm{M})$. Data are representative of three independent experiments. Percentage of e) annexin V-positive and f) PI-positive primary CF bronchial epithelial cells was assessed at $24 \mathrm{~h}$ after AZM treatment and in untreated cells. Data are presented as median (range) of three independent experiments. Statistical analysis was performed with the paired nonparametric Wilcoxon rank test. IM: infection medium; Ann+: annexin V positive control (1 $\mu \mathrm{g} \cdot \mathrm{mL}^{-1}$ polyinosinic:polycytidylic acid); PI+: PI positive control (frozen cells).

\section{Azithromycin increases RV1B surface receptor and PRRs in RV1B-infected CF bronchial epithelial cells}

To assess whether an increase in virus entry and sensing contributes to the antiviral properties of azithromycin, the effect of azithromycin treatment on the expression of the RV1B surface receptor (LDLR) and PRRs involved in the recognition of RVs, such as TLR3, TLR2, RIG-I and MDA5 [24, 29], was determined at $24 \mathrm{~h}$ post-infection (fig. 4). We found that the constitutive mRNA levels of LDLR, TLR3, TLR2, RIG-I and MDA5 were not modified by the azithromycin treatment. However, we observed a 

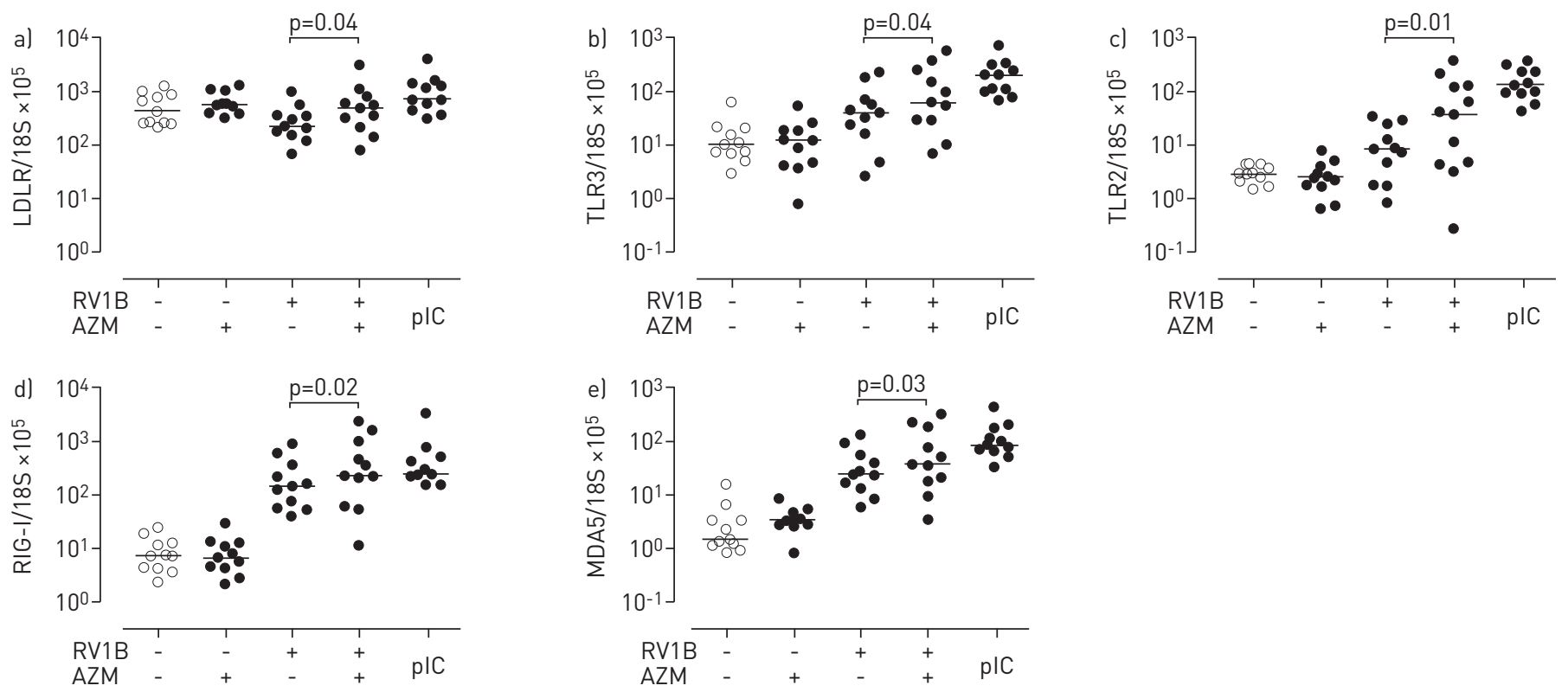

FIGURE 4 Azithromycin (AZM) stimulates the expression of rhinovirus (RV)1B surface receptor and pattern recognition receptors in primary cystic fibrosis (CF) bronchial epithelial cells. mRNA levels of a) low density lipoprotein receptor (LDLR), b) Toll-like receptor (TLR)3, c) TLR2, d) retinoic acid-inducible gene (RIG)-I and e) melanoma differentiation-associated protein (MDA) 5 were measured at $24 \mathrm{~h}$ after RV1B infection by real-time quantitative PCR in primary CF bronchial epithelial cells treated or not treated with AZM $(50 \mu \mathrm{M})$. Expression levels were normalised to those of 18S ribosomal RNA (18S). Data are presented as median of 11 independent experiments. Statistical analysis was performed with the paired nonparametric Wilcoxon rank test. pIC: polyinosinic: polycytidylic acid $\left(1 \mu \mathrm{g} \cdot \mathrm{mL}^{-1}\right)$.

significantly 2.2-fold increased expression level of the RV1B cell surface entry receptor LDLR by treatment with azithromycin in RV1B-infected CF bronchial cells compared with untreated cells $(p=0.04)$. Similarly, AZM treatment significantly upregulated the RV1B-induced expression levels of the TLR3, TLR2, RIG-I and MDA5 in CF bronchial cells compared to untreated cells (1.5-, 4.3-, 1.6- and 1.6-fold increase, respectively).

\section{Azithromycin amplifies RV1B-induced IFN and ISG responses in CF bronchial epithelial cells}

To examine whether the antiviral effect of azithromycin in CF bronchial cells is associated with the induction of IFNs and subsequent ISG transcription, we assessed RV1B-induced mRNA and/or protein levels of type I and III IFNs (IFN- $\beta$ and IFN- $\lambda 2 / 3$, respectively) and ISGs (viperin, MxA and OAS) before and after azithromycin treatment at $24 \mathrm{~h}$ post-infection. We observed that azithromycin significantly decreased the basal level of MxA; however, its expression level was close to the detection limit of the PCR assay (Ct values $>35$ ) and, therefore, these data have to be interpreted with caution. We found that RV1B-induced mRNA levels of IFN- $\lambda 2 / 3$ were 2.2 -fold significantly increased $(p=0.03)$ in CF bronchial cells compared with untreated cells (fig. $5 \mathrm{a}$ ). There was also a trend for increased IFN- $\beta$ mRNA levels after azithromycin treatment (fig. 5b). Similarly, we observed that azithromycin treatment upregulated RV1B-induced IFN- $\beta$ protein levels 1.2-fold in supernatants of CF bronchial cells compared with untreated cells ( $\mathrm{p}=0.007$; fig. $5 \mathrm{c}$ ). RV1B-induced mRNA levels of the ISGs viperin and MxA were also upregulated by azithromycin treatment (4.2- and 1.4-fold, respectively) in CF bronchial cells compared with untreated cells ( $p=0.003$ and $p=0.02$, respectively; fig. $5 \mathrm{~d}$ and e). In contrast, RV1B-induced mRNA level of OAS remained unaffected by azithromycin treatment (fig. $5 \mathrm{f}$ ).

\section{Azithromycin does not prevent RV1B-induced pro-inflammatory cytokine production in CF bronchial epithelial cells}

There are controversial results regarding whether azithromycin has in vitro anti-inflammatory effects in $\mathrm{CF}$ airway epithelial cells [12, 30-32]. To study whether anti-inflammatory properties of azithromycin potentially underlie its antiviral effect, we assessed the effects of azithromycin on the inflammatory response in primary CF bronchial cells infected with RV1B. We measured the production of IL-8, IL-6 and IP-10 at both the mRNA level (fig. $6 \mathrm{a}-\mathrm{c}$ ) by qPCR and at the protein level (fig. $6 \mathrm{~d}-\mathrm{f}$ ) by ELISA. We found that azithromycin treatment neither reduced RV1B-induced mRNA levels nor decreased RV1B-induced protein levels of IL-8 and IL-6 in CF bronchial cells compared with untreated cells. Interestingly, azithromycin treatment significantly increased the mRNA level of RV1B-induced IP-10 by 

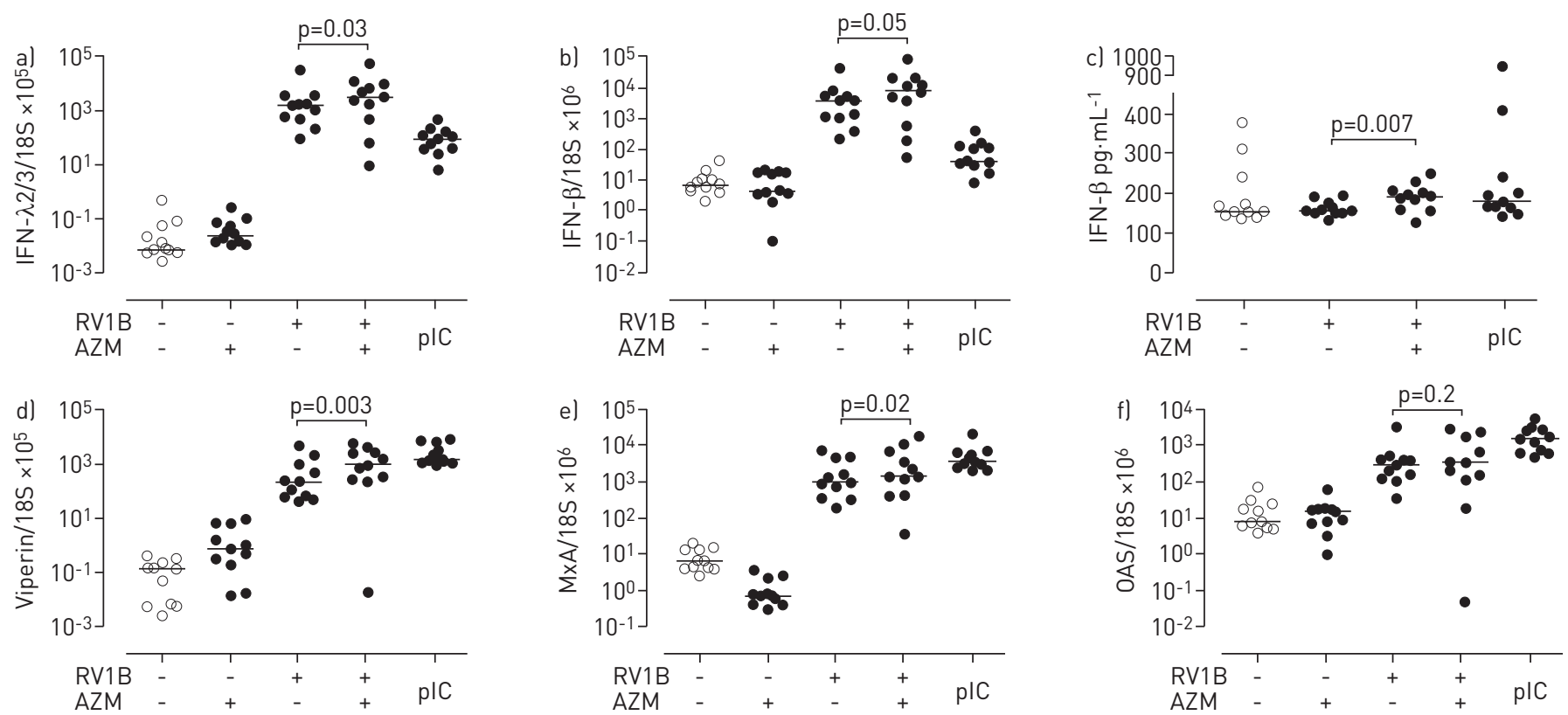

FIGURE 5 Azithromycin (AZM) upregulates interferons (IFNs) and IFN-stimulated genes (ISGs) in primary cystic fibrosis (CF) bronchial epithelial cells. mRNA levels of a) IFN- $\lambda 2 / 3$ and b) IFN- $\beta$, and c) protein levels of IFN- $\beta$ were measured $24 \mathrm{~h}$ after rhinovirus (RV) $1 \mathrm{~B}$ infection by real-time quantitative PCR (qPCR) or ELISA in primary CF bronchial epithelial cells treated or not treated with AZM $(50 \mu \mathrm{M})$. mRNA levels of the ISGs d) viperin (virus inhibitory protein, endoplasmic reticulum-associated, IFN inducible), e) MxA and f) $2^{\prime}, 5^{\prime}$-oligoadenylate synthase (OAS) were measured $24 \mathrm{~h}$ after RV1B infection by real-time qPCR in primary CF bronchial epithelial cells treated or not treated with AZM $(50 \mu \mathrm{M})$. Expression levels were normalised to those of $18 \mathrm{~S}$ ribosomal RNA (18S). Data are presented as median of 11 independent experiments. Statistical analysis was performed with the paired non-parametric Wilcoxon rank test. pIC: polyinosinic:polycytidylic acid $\left(1 \mu \mathrm{g} \cdot \mathrm{mL}^{-1}\right)$.

2.2-fold ( $\mathrm{p}=0.01$; fig. $6 \mathrm{c}$ ) and a modest but not statistically significant increase of RV1B-induced IP-10 protein level in azithromycin-treated CF bronchial cells was observed (fig. 6f).

\section{Discussion}

A recent study by Gielen et al. [12] has shown that azithromycin, a macrolide antibiotic commonly used to treat CF patients, has promising effects against RV infection in vitro in human bronchial epithelial cells derived from healthy subjects. Therefore, we comprehensively studied the relevance of azithromycin as a possible treatment to control the increased viral replication observed in CF airway epithelial cells infected with RV. Confirming our hypothesis of an antiviral effect of azithromycin, we found that azithromycin decreased viral replication in CF bronchial cells, possibly by inducing an early antiviral response mediated by the induction of PRRs, IFNs and ISGs. Azithromycin did not act by inhibiting the production of pro-inflammatory cytokines. Thus, the antiviral properties of azithromycin may provide an explanation for the favourable effects of long-term macrolide treatment observed in CF patients.

In accordance with previous reports $[18,20,28]$, we found that CF bronchial cells revealed an increased viral replication compared to controls. Previously, SUTANTO et al. [20] reported that the viral copy number in CF airway epithelial cells in vitro infected with RV1B is higher than in non-CF cells. Similarly, ZHENG et al. [28] identified increased in vitro viral replication in CF airway epithelial cells compared with non-CF cells infected with human parainfluenza virus 3. Interestingly, increased RV replication in CF has been confirmed in an ex vivo model using bronchoalveolar lavages from CF and control patients [18]. In our study, we further demonstrated that azithromycin treatment had antiviral properties characterised by a seven-fold reduced RV1B replication in CF bronchial cells. Our findings confirm previous observations showing antiviral properties of macrolides such as azithromycin [12], erythromycin [10], clarithromycin [9] and bafilomycin A1 [11] in RV-infected airway epithelial cells. In addition, we observed that the decreased RV1B load after azithromycin treatment was not attributable to azithromycin-induced cytotoxicity.

We found that the antiviral action of azithromycin in CF bronchial cells is associated with the stimulation of antiviral mechanisms through the increase of IFNs, ISGs and PRRs such as TLR2/3, RIG-I and MDA5. The antiviral activity of azithromycin is, surprisingly, associated with a slightly increased expression of the minor-group RV surface receptor LDLR in CF bronchial cells. This observation is novel and intriguing. Several studies identified a decreased virus entry characterised by a reduced expression of the intercellular 

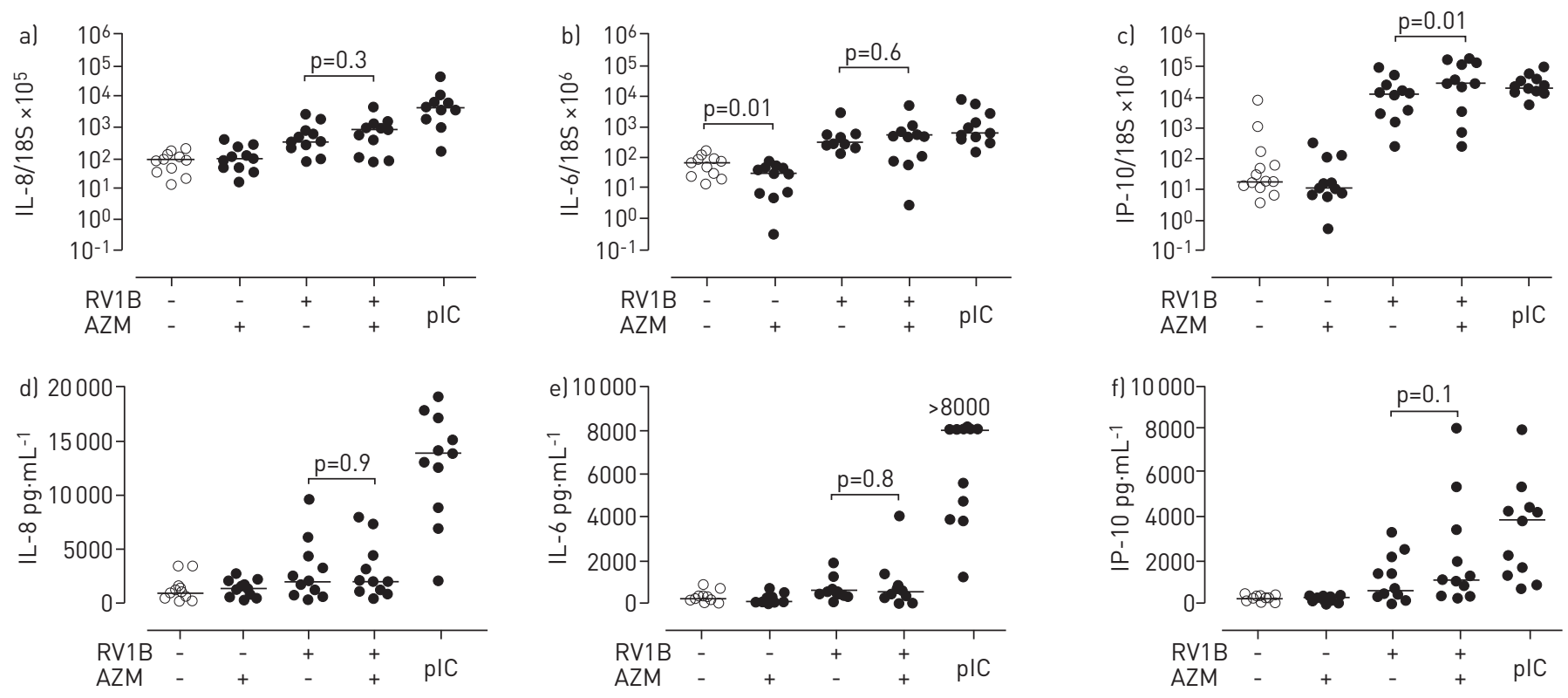

FIGURE 6 Azithromycin (AZM) does not prevent rhinovirus (RV)1B-induced pro-inflammatory cytokine production in primary cystic fibrosis (CF) bronchial epithelial cells. $a-c)$ mRNA levels in cell lysates and d-f) protein levels in supernatants of the pro-inflammatory cytokines interleukin (IL)-8 (a and d), IL-6 (b and e) and the $10-\mathrm{kDa}$ interferon- $\gamma$-induced protein (IP-10) ( $\mathrm{c}$ and $\mathrm{f}$ ) were measured before and $24 \mathrm{~h}$ after RV1B infection by real-time quantitative PCR or ELISA respectively in primary CF bronchial epithelial cells treated or not treated with azithromycin $(50 \mu \mathrm{M})$. Expression levels were normalised to those of $18 \mathrm{~S}$ ribosomal RNA (18S). Data are presented as median of 11 independent experiments. Statistical analysis was done with the paired non-parametric Wilcoxon rank test. pIC: polyinosinic:polycytidylic acid $\left(1 \mu \mathrm{g} \cdot \mathrm{mL}^{-1}\right)$.

cell adhesion molecule 1, which is the cell surface entry receptor for the major group of RVs, as a possible anti-RV mechanism of macrolide treatment [9-11]. Interestingly, this suggests that macrolides have antiviral effects on both minor- and major-type RV infection independently of the expression of the respective surface entry receptor. Indeed, previous studies showed that augmentation of antiviral activity was not dependent on RV serotype [12]. In contrast to our findings, SuzuKi et al. [10] showed decreased minor-type RV replication despite unmodified expression of LDLR by macrolide treatment. The discrepancy of our observations with these data might be explained by different macrolides used (azithromycin versus erythromycin), different duration of macrolide pre-treatment ( $24 \mathrm{~h}$ versus 3 days), different concentrations (50 versus $10 \mu \mathrm{M}$ ), different cell sources (bronchial versus tracheal epithelial cells) or different strains of minor-type RV used (RV1B versus RV2).

It is conceivable that the increased patterning of RV through PRRs, induced by azithromycin treatment, amplifies IFN responses leading to the expression of ISGs, the mediators of the antiviral response. The ISG viperin, which we found to be increased in CF bronchial cells upon treatment with azithromycin, has recently been demonstrated to be critical in the host response to RV infection [33]. Our results of an enhanced early innate immune response mediated by increased expression of IFNs and ISGs by azithromycin provide a putative explanation for the antiviral activity of azithromycin in CF bronchial cells.

Primary CF bronchial cells have an elevated inflammatory response upon virus infection in comparison to healthy cells $[20,28]$. Therefore, we evaluated the potential of azithromycin as a modifier of the inflammatory response of CF bronchial cells. Our results demonstrated that while stimulating the antiviral response, azithromycin treatment did not significantly prevent RV-induced expression of pro-inflammatory cytokines such as IL-8 and IL-6 in RV1B-infected CF bronchial cells. Interestingly, we observed an increased expression of RV1B-induced IP-10 by azithromycin. In contrast to pro-inflammatory cytokines, which are under the control of the transcription factor NF- $\mathrm{kB}$ (nuclear factor, K-light chain enhancer of activated B-cells) [34], the transcription of the IP-10 gene is dependent on NF- $\mathrm{KB}$ and the IFNs pathway by means of IFN-regulatory factors $[16,35,36]$. These results are in line with our observation of increased expression of RV1B-induced IFNs by azithromycin treatment in the CF cells. The following limitations of our study should be taken into account when interpreting the data. First, we used an in vitro model based on primary submerged epithelial cell cultures grown as monolayer. Thus, the use of fully differentiated airway epithelial cells cultured at the air-liquid interface might be needed to more accurately mimic the in vivo situation. Secondly, synergisms between bacteria and RV have not been evaluated. Indeed, the microbiological environment present in the lung of study subjects, such as 
$P$. aeruginosa colonisation, has been claimed to modify the antiviral response to RV infection [37]. However, viral load between bronchial cells from CF children that were P. aeruginosa positive at the time of sampling and bronchial cells derived from CF children free of $P$. aeruginosa did not differ (data not shown). Interestingly, a recent study demonstrated that bacterial detection and the presence of pulmonary exacerbations in CF patients were not associated [38]. Moreover, we sampled only at $24 \mathrm{~h}$ after infection. Greater effects may be seen at later time points of sampling. Furthermore, we did not perform nasal swabbing to detect ongoing concomitant RV infection in the upper airways.

In our study, however, we focused on bronchial epithelial cells, the primary site of CF lung disease and the main target for RVs in the lower airways. Indeed, RV infection in CF causes a high RV burden in the lower airways [18] and viral infections of the lower airways of CF patients lead to a risk of hospitalisation and to persistent deterioration of lung function parameters, and are related to increased lower respiratory tract morbidity in infants with CF $[39,40]$. Thus, cells from the lower respiratory tract are more relevant to $\mathrm{RV}$-associated respiratory tract morbidity of $\mathrm{CF}$ infants than epithelial cells from the upper respiratory tract. However, as other cells such as neutrophils and alveolar macrophages play a pivotal role in CF airway disease pathogenesis, we cannot exclude a bronchial epithelial cell-independent role of azithromycin, especially in the context of possible anti-inflammatory properties of azithromycin. Indeed, a recent study shows that azithromycin reduces the pro-inflammatory cytokine production of M1-polarised alveolar macrophages in CF mice [41], and decreases pro-inflammatory cytokines expression such as IL-8 or tumour necrosis factor- $\alpha$ by human alveolar macrophages of CF [42] and healthy subjects [43]. Furthermore, macrolides have been shown to reduce the activation of neutrophils and to decrease neutrophil chemotaxis to the site of infection in the airways [44].

In summary, we identified antiviral properties of azithromycin on RV infection in primary bronchial epithelial cells obtained from CF patients. Our data suggest that the mechanism of action of azithromycin is mediated by a global amplification of the IFN pathway-mediated antiviral responses through the induction of PRRs, IFNs and ISGs leading to a reduction of RV replication.

Since there is a lack of treatment option for virus-induced pulmonary exacerbation in patients with CF, our study suggests that clinical studies are needed to further elucidate the potential role of azithromycin in the management and prevention of RV-induced exacerbations of CF lung disease, which could be of relevance especially to CF infants and children.

\section{Acknowledgements}

The authors would like to thank all study participants and their families.

\section{References}

1 Asner $\mathrm{S}$, Waters $\mathrm{V}$, Solomon $\mathrm{M}$, et al. Role of respiratory viruses in pulmonary exacerbations in children with cystic fibrosis. J Cyst Fibros 2012; 11: 433-439.

2 Wat D, Gelder C, Hibbitts S, et al. The role of respiratory viruses in cystic fibrosis. J Cyst Fibros 2008; 7: 320-328.

3 de Almeida MB, Zerbinati RM, Tateno AF, et al. Rhinovirus $\mathrm{C}$ and respiratory exacerbations in children with cystic fibrosis. Emerg Infect Dis 2010; 16: 996-999.

4 Flight WG, Bright-Thomas RJ, Tilston P, et al. Incidence and clinical impact of respiratory viruses in adults with cystic fibrosis. Thorax 2014; 69: 247-253.

5 Giebels K, Marcotte JE, Podoba J, et al. Prophylaxis against respiratory syncytial virus in young children with cystic fibrosis. Pediatr Pulmonol 2008; 43: 169-174.

6 Renk H, Regamey N, Hartl D. Influenza A(H1N1)pdm09 and Cystic Fibrosis Lung Disease: A Systematic Meta-Analysis. PLoS One 2014; 9: e78583.

7 Flume PA, Mogayzel PJ Jr, Robinson KA, et al. Cystic fibrosis pulmonary guidelines: treatment of pulmonary exacerbations. Am J Respir Crit Care Med 2009; 180: 802-808.

8 Southern KW, Barker PM, Solis A. Macrolide antibiotics for cystic fibrosis. Cochrane Database Syst Rev 2004; 2: CD002203.

9 Jang YJ, Kwon HJ, Lee BJ. Effect of clarithromycin on rhinovirus-16 infection in A549 cells. Eur Respir J 2006; 27 : $12-19$.

10 Suzuki T, Yamaya M, Sekizawa K, et al. Erythromycin inhibits rhinovirus infection in cultured human tracheal epithelial cells. Am J Respir Crit Care Med 2002; 165: 1113-1118.

11 Suzuki T, Yamaya M, Sekizawa $\mathrm{K}$, et al. Bafilomycin $\mathrm{A}_{1}$ inhibits rhinovirus infection in human airway epithelium: effects on endosome and ICAM-1. Am J Physiol Lung Cell Mol Physiol 2001; 280: L1115-L1127.

12 Gielen V, Johnston SL, Edwards MR. Azithromycin induces anti-viral responses in bronchial epithelial cells. Eur Respir J 2010; 36: 646-654.

13 Vareille M, Kieninger E, Edwards MR, et al. The airway epithelium: soldier in the fight against respiratory viruses. Clin Microbiol Rev 2011; 24: 210-229.

14 Khaitov MR, Laza-Stanca V, Edwards MR, et al. Respiratory virus induction of alpha-, beta- and lambda-interferons in bronchial epithelial cells and peripheral blood mononuclear cells. Allergy 2009; 64: 375-386.

15 Becker TM, Durrani SR, Bochkov YA, et al. Effect of exogenous interferons on rhinovirus replication and airway inflammatory responses. Ann Allergy Asthma Immunol 2013; 111: 397-401.

16 Bartlett NW, Slater L, Glanville N, et al. Defining critical roles for NF- $\kappa \mathrm{B}$ p65 and type I interferon in innate immunity to rhinovirus. EMBO Mol Med 2012; 4: 1244-1260. 
17 Cakebread JA, Xu Y, Grainge C, et al. Exogenous IFN- $\beta$ has antiviral and anti-inflammatory properties in primary bronchial epithelial cells from asthmatic subjects exposed to rhinovirus. J Allergy Clin Immunol 2011; 127: $1148-1154$.

18 Kieninger E, Singer F, Tapparel C, et al. High rhinovirus burden in lower airways of children with cystic fibrosis. Chest 2013; 143: 782-790.

19 McNamara PS, Kicic A, Sutanto EN, et al. Comparison of techniques for obtaining lower airway epithelial cells from children. Eur Respir J 2008; 32: 763-768.

20 Sutanto EN, Kicic A, Foo CJ, et al. Innate inflammatory responses of pediatric cystic fibrosis airway epithelial cells: effects of nonviral and viral stimulation. Am J Respir Cell Mol Biol 2011; 44: 761-767.

21 Papi A, Johnston SL. Rhinovirus infection induces expression of its own receptor intercellular adhesion molecule 1 (ICAM-1) via increased NF-кB-mediated transcription. J Biol Chem 1999; 274: 9707-9720.

22 Di Paolo A, Barbara C, Chella A, et al. Pharmacokinetics of azithromycin in lung tissue, bronchial washing, and plasma in patients given multiple oral doses of 500 and $1000 \mathrm{mg}$ daily. Pharmacol Res 2002; 46: 545-550.

23 Zhao X, Ai M, Guo Y, et al. Poly I:C-induced tumor cell apoptosis mediated by pattern-recognition receptors. Cancer Biother Radiopharm 2012; 27: 530-534.

24 Slater L, Bartlett NW, Haas JJ, et al. Co-ordinated role of TLR3, RIG-I and MDA5 in the innate response to rhinovirus in bronchial epithelium. PLoS Pathog 2010; 6: e1001178.

25 Contoli M, Message SD, Laza-Stanca V, et al. Role of deficient type III interferon-lambda production in asthma exacerbations. Nat Med 2006; 12: 1023-1026.

26 Llorente-Cortes V, Otero-Vinas M, Sanchez S, et al. Low-density lipoprotein upregulates low-density lipoprotein receptor-related protein expression in vascular smooth muscle cells: possible involvement of sterol regulatory element binding protein-2-dependent mechanism. Circulation 2002; 106: 3104-3110.

27 Pivarcsi A, Bodai L, Rethi B, et al. Expression and function of Toll-like receptors 2 and 4 in human keratinocytes. Int Immunol 2003; 15: 721-730.

28 Zheng S, De BP, Choudhary S, et al. Impaired innate host defense causes susceptibility to respiratory virus infections in cystic fibrosis. Immunity 2003; 18: 619-630.

29 Wang Q, Nagarkar DR, Bowman ER, et al. Role of double-stranded RNA pattern recognition receptors in rhinovirus-induced airway epithelial cell responses. J Immunol 2009; 183: 6989-6997.

30 Cigana C, Assael BM, Melotti P. Azithromycin selectively reduces tumor necrosis factor alpha levels in cystic fibrosis airway epithelial cells. Antimicrob Agents Chemother 2007; 51: 975-981.

31 Cigana C, Nicolis E, Pasetto M, et al. Anti-inflammatory effects of azithromycin in cystic fibrosis airway epithelial cells. Biochem Biophys Res Commun 2006; 350: 977-982.

32 Saint-Criq V, Ruffin M, Rebeyrol C, et al. Azithromycin fails to reduce inflammation in cystic fibrosis airway epithelial cells. Eur J Pharmacol 2012; 674: 1-6.

33 Proud D, Turner RB, Winther B, et al. Gene expression profiles during in vivo human rhinovirus infection: insights into the host response. Am J Respir Crit Care Med 2008; 178: 962-968.

34 Matsusaka T, Fujikawa K, Nishio Y, et al. Transcription factors NF-IL6 and NF-kappa B synergistically activate transcription of the inflammatory cytokines, interleukin 6 and interleukin 8. Proc Natl Acad Sci USA 1993; 90: 10193-10197.

35 Zaheer RS, Proud D. Human rhinovirus-induced epithelial production of CXCL10 is dependent upon IFN regulatory factor-1. Am J Respir Cell Mol Biol 2010; 43: 413-421.

36 Taima K, Imaizumi T, Yamashita K, et al. Expression of IP-10/CXCL10 is upregulated by double-stranded RNA in BEAS-2B bronchial epithelial cells. Respiration 2006; 73: 360-364.

37 Chattoraj SS, Ganesan S, Faris A, et al. Pseudomonas aeruginosa suppresses interferon response to rhinovirus infection in cystic fibrosis but not in normal bronchial epithelial cells. Infect Immun 2011; 79: 4131-4145.

38 Stelzer-Braid S, Johal H, Skilbeck K, et al. Detection of viral and bacterial respiratory pathogens in patients with cystic fibrosis. J Virol Methods 2012; 186: 109-112.

39 Hiatt PW, Grace SC, Kozinetz CA, et al. Effects of viral lower respiratory tract infection on lung function in infants with cystic fibrosis. Pediatrics 1999; 103: 619-626.

40 van Ewijk BE, van der Zalm MM, Wolfs TF, et al. Prevalence and impact of respiratory viral infections in young children with cystic fibrosis: prospective cohort study. Pediatrics 2008; 122: 1171-1176.

41 Meyer M, Huaux F, Gavilanes X, et al. Azithromycin reduces exaggerated cytokine production by M1 alveolar macrophages in cystic fibrosis. Am J Respir Cell Mol Biol 2009; 41: 590-602.

42 Cory TJ, Birket SE, Murphy BS, et al. Impact of azithromycin treatment on macrophage gene expression in subjects with cystic fibrosis. J Cyst Fibros 2014; 13: 164-171.

43 Kurdowska A, Noble JM, Griffith DE. The effect of azithromycin and clarithromycin on ex vivo interleukin-8 (IL-8) release from whole blood and IL-8 production by human alveolar macrophages. J Antimicrob Chemother 2001; 47: 867-870.

44 Tamaoki J, Sakai N, Tagaya E, et al. Macrolide antibiotics protect against endotoxin-induced vascular leakage and neutrophil accumulation in rat trachea. Antimicrob Agents Chemother 1994; 38: 1641-1643. 